INPLASY

PROTOCOL

To cite: Cai et al. Zhisou

Powder in treatment of postinfectious cough: $A$ protocol for systematic review and meta-analysis. Inplasy protocol 202090096. doi: 10.37766/inplasy2020.9.0096

Received: 26 September 2020

Published: 27 September 2020

Corresponding author:

Weihong Li

Iwh@cdutcm.edu.cn

Author Affiliation:

Basic Medical College, Chengdu University of TCM

Support: NSFC.

Review Stage at time of this submission: Preliminary searches.

Conflicts of interest:

The authors have no conflicts of interest to disclose.

\section{Zhisou Powder in treatment of postinfectious cough: A protocol for} systematic review and meta-analysis

Cai, HYㅜㄴ Li, WH²; Kang, SX3; He, J4; Yu, P5; Li, H6.

Review question / Objective: We aim to systematically review the effectiveness and safety of Zhisou Powder For postinfectious cough.

Condition being studied: Postinfectious cough is a common disease in primary care affecting. approximately $40 \%$ of adults develop a postinfectious cough after an acute respiratory tract infection. it seriously affects the quality of life of patients. However, the effects of conventional medication such as corticosteroids, ipratropium, dextromethorphan, antihistamines are not satisfactory. Clinical studies have found that Zhisou Powder has a significant effect on postinfectious cough. the purpose of this study is to provide high-quality evidence on the efficacy and safety of Zhisou Powder in treating postinfectious cough.

INPLASY registration number: This protocol was registered with the International Platform of Registered Systematic Review and Meta-Analysis Protocols (INPLASY) on 27 September 2020 and was last updated on 27 September 2020 (registration number INPLASY202090096).

\title{
INTRODUCTION
}

Review question / Objective: We aim to systematically review the effectiveness and safety of Zhisou Powder For postinfectious cough.
Condition being studied: Postinfectious cough is a common disease in primary care affecting. approximately $40 \%$ of adults develop a postinfectious cough after an acute respiratory tract infection. it seriously 
affects the quality of life of patients. However, the effects of conventional medication such as corticosteroids, ipratropium, dextromethorphan, antihistamines are not satisfactory. Clinical studies have found that Zhisou Powder has a significant effect on postinfectious cough. the purpose of this study is to provide high-quality evidence on the efficacy and safety of Zhisou Powder in treating postinfectious cough.

\section{METHODS}

Participant or population: Participants who have been diagnosed with postinfectious cough will be included.

Intervention: The experimental group only used Zhisou Powder or combined with interventions of the control group.

Comparator: The control group used placebo control or no Treatment or conventional medication, such as corticosteroids, ipratropium, dextromethorphan, antihistamines.

Study designs to be included: All randomized controlled trials(RCTs) about Zhisou Powder for postinfectious cough will be included regardless of language.

Eligibility criteria: All randomized controlled trials(RCTs) about Zhisou Powder (ZP) for postinfectious cough (PIC) will be included regardless of language. The following studies: case series, quasi-RCTs Case reports, non-RCTs, cell experiments, animal experiments will be excluded. Participants:Participants who have been diagnosed with PIC will be included and regardless of gender, age, ethnicity, economic status, or restrictions, educational. Types of interventions:The experimental group only used ZP or combined with interventions of the control group. The control group used placebo control or no Treatment or conventional medication, such as corticosteroids, ipratropium, dextromethorphan, antihistamines.
Information sources: The following electronic databases will be comprehensively searched including: PubMed, Cochrane Library, EMBASE, MEDLINE, CNKI, CBM, VIP, and WAN FANG. all the literature retrieved is from the time when the database establishment to 20 May 2020.The missing information will be supplemented by contacting the original author.

Main outcome(s): The primary outcomes were cough relief rate and cough resolution rate.

Quality assessment / Risk of bias analysis: The Cochrane Collaboration's tool will be used to assess the risk of literature bias. The methodological quality will be assessed by two investigators independently using RevMan 5.3.0. The following seven aspects will be evaluated. Including: random, blinding of participants and investigators, sequence generation, allocation concealment, the blindness of outcome assessments, selective outcome reporting, incomplete outcome data, and other biases. As a result, every included study will be assessed as low, unclear, or high bias.

Strategy of data synthesis: For data analysis, RevMan 5.3.0 that is provided by the Cochrane Collaboration will be used. we will use the chi-square test and 12 statistic to evaluate the heterogeneity of similar studies. If $P \geq 0.05$ and $12 \leq 50 \%$, we believe it is low heterogeneity. As result, we will use a fixed-effects model. If $\mathrm{P}<0.05$ and $12>50 \%$, it means there is heterogeneity. we will use a random-effects model. For the enumeration data, odds ratio(OR) with a $95 \%$ confidence interval (CI) will be used to represent. We will use mean difference (MD) with $95 \% \mathrm{Cl}$ to express the measurement data. The statistical significant difference is thought of as $P<0.05$.

Subgroup analysis: If the studies show significant heterogeneity. subgroup analysis will be performed to explore the source of heterogeneity. 
Sensibility analysis: Furthermore if necessary, a sensitivity analysis will be performed.

Country(ies) involved: China.

Keywords: Zhisou Powder, effectiveness and safety; postinfectious cough; systematic review.

Contributions of each author:

Author 1 - Haiyang Cai - Conceptualization; Methodology; Project administration; Writing - original draft; Writing - review \& editing.

Author 2 - Weihong Li - Conceptualization; Funding acquisition; Project administration; Validation.

Author 3 - Shixin Kang - Conceptualization; Investigation; Methodology; Writing original draft; Writing - review \& editing.

Author 4 - Jing He - Software.

Author 5 - Peng Yu - Data curation.

Author 6 - Han Li - Supervision. 